\title{
THE ISRAEL EMS STROKE PRE-NOTIFICATION SMARTPHONE APP A NEW TOOL TO INVESTIGATE PREHOSPITAL TRIAGE ACCURACY
}

\author{
N.Horrany 1 , M.Lynn, M.Abulafi ${ }_{1}$, J.Cohen ${ }_{1}$, N.Kaufmann 1 , N.M.Bornstein 1 , R. Strugo 2 , H. \\ Karadi $_{2}$ O. Blushteinl, Rosenblat, R.Eichel 1 \\ 1 Stroke Unit, Shaare Zedek Medical Center, Jerusalem \\ 2Magen David Adom, National Israeli EMS
}

Background:

Prehospital Triage and Pre-notification Systems for Acute Stroke (AS) patients are the most important parameters to increase the likelihood of AS patients to receive reperfusion therapy when arriving at the hospital. The Face Arm Speech Test (FAST) is the major tool today for Emergency Medical Services (EMS) to apply such a triage in the prehospital setting. Even though FAST is the most used tool today for EMS, it is unclear what is the contribution of each part of FAST to identify Stroke in the prehospital setting, and if other parameters could be of help to improve the triage of paramedics in the field.

\section{Objective:}

We attempted to investigate the triage accuracy of the newly developed Stroke Pre-notification Smartphone App (SPSA) for the Israeli national EMS (Magen David Adom) teams.

\section{Methods:}

We retrospectively analyzed our institution based stroke registry between 07/2016 July/2017 for all patients arriving for suspected stroke to our hospital. We compared patients arriving with AS with prehospital triage by using the smartphone app with those without usage of the app. We then compared, in all patient arriving with prior use of SPSA, the accuracy of clinical parameters like Mean Arterial Pressure (MAP), pulse, breathing, forced eye deviation and each part of the FAST to be able to distinguish between AS patients and those with other final diagnosis.

\section{Stroke Pre-notification Smartphone App}
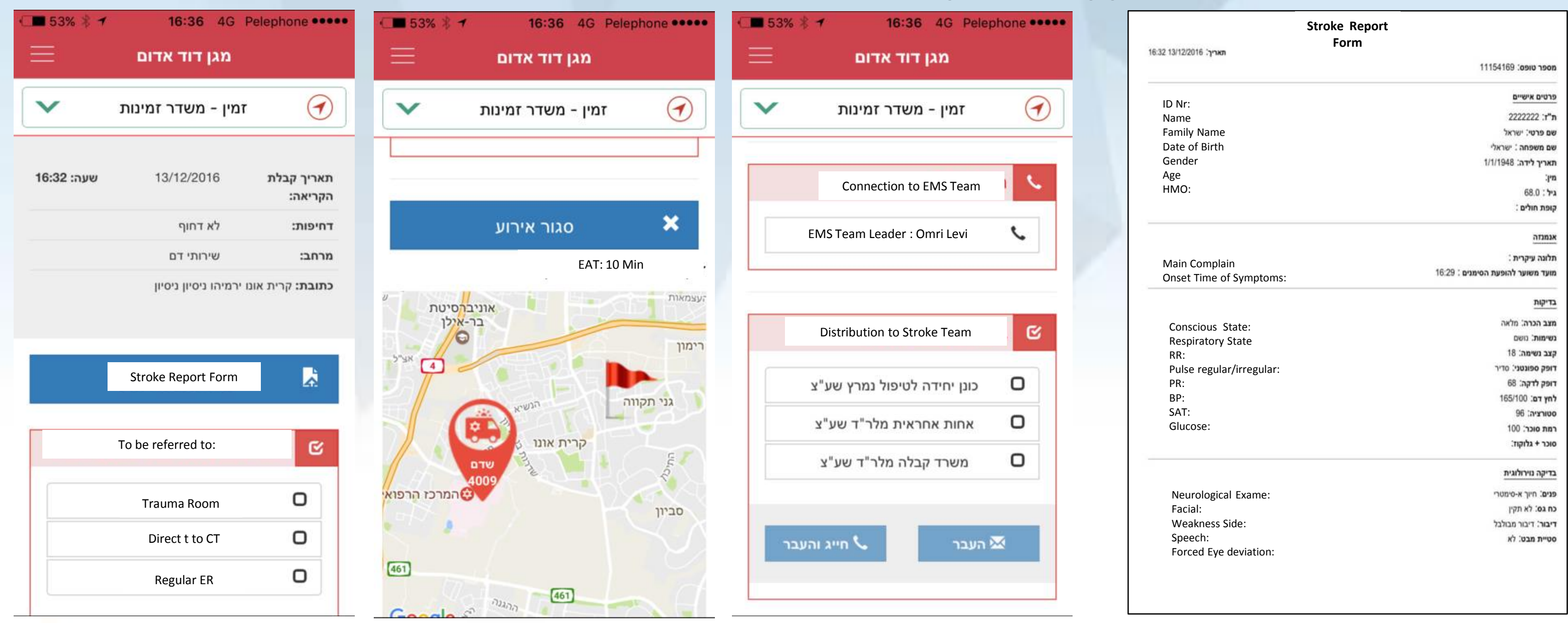

\section{Conclusion:}

Prehospital triage is not only a major contributor of increasing the chance of AS patients to receive fast treatment but also the most important tool to manage a high volume of suspected AS patients and to be differentiate them between real AS patients and mimickers even before arriving to the hospital. Our Data shows the first time that the classical FAST triage system might have to be newly evaluated and adapted with subtracting and adding other clinical features to make the triage more accurate. 